\title{
PUBLICACIÓN DE LOS TRABAJOS PRESENTADOS A LOS CONGRESOS CIENTIIFICOS DE ESTUDIANTES DE MEDICINA, PERÚ 2002-2009: CARACTERÍSTICAS Y FACTORES ASOCIADOS
}

\author{
Luis Miguel Toro-Polo1,2,a, Reneé Pereyra-Elíase,2,a, Ayar Nizama-Vía 1,2,a, Luis Fernando Ng-Sueng',2,a, \\ Eduardo Vélez-Segovia ${ }^{1,2, a}$, Edén Galán-Rodas ${ }^{3, b}$, Percy Mayta-Tristán ${ }^{1, b}$
}

RESUMEN

Objetivos. Determinar la proporción de publicación de los resúmenes presentados a los congresos científicos nacionales
de estudiantes de medicina de Perú entre los años 2002 y 2009 y sus factores asociados. Materiales y métodos. Se
realizó una cohorte retrospectiva, se evaluó las características de todos los resúmenes presentados y se determinó si
habían sido publicados en revistas científicas utilizando una estrategia de búsqueda validada con Google Académico. Se
calculó los riesgos relativos (RR), crudos y ajustados mediante la regresión de Poisson con varianza robusta para evaluar
asociación con los factores analizados. Resultados. Se analizó 532 resúmenes; $52(9,8 \%$ ) fueron publicados en revistas
científicas luego de su participación en el congreso; en todos los casos fue en revistas peruanas y en español. La principal
revista donde se publicaron fue (CIMEL) (13/52). La mediana del tiempo de publicación fue de 13 meses (rango: $0-75)$.
El que un alumno de la Universidad Nacional Mayor de San Marcos (RR: 5,$18 ;$ IC95\%:2,3-11,6) sea autor del resumen y
que el resumen sea de autoría colaborativa entre alumnos de dos universidades (RR: 3,$64 ;$ IC95\%:1,1-11,7) son factores
asociados en el análisis multivariado. Conclusiones. La proporción de publicación de resúmenes presentados a los
congresos científicos nacionales de estudiantes de medicina de Perú es baja. Se deben implementar nuevas medidas y
reforzar las existentes para incentivar una mayor publicación de los trabajos presentados.

Palabras clave: Investigación; Congresos; Resúmenes de reuniones; Publicaciones científicas y técnicas; Estudiantes de Medicina (fuente: DeCS BIREME).

\section{PUBLICATION OF SUMMARIES PRESENTED AT THE SCIENTIFIC CONGRESSES OF MEDICAL STUDENTS, PERU 2002-2009: CHARACTERISTICS AND RELATED FACTORS}

\section{ABSTRACT}

\begin{abstract}
Objectives. To determine the publication rate of abstracts submitted at the national scientific meetings of medical students in Peru between 2002 and 2009, and associated factors. Materials and methods. A retrospective cohort was performed; the characteristics of overall abstract submitted were assessed. In addition, whether they were published in scientific journals was determined by way of a search strategy validated using Google Scholar. Crude and adjusted relative risks $(\mathrm{RR})$ were calculated using Poisson regression with robust variance to assess association with the analyzed factors. Results. 532 abstracts were analyzed, 52 (9.8\%) of which were published in scientific journals after their presentation at a scientific meeting. All of them were published in Peruvian journals written in Spanish. The most important journal in which they were published was Ciencia e Investigación Médica Estudiantil Latinoamericana (CIMEL) (13/52). The median of publication time was 13 months (range: 0-75). The fact that a student from the Universidad Nacional Mayor de San Marcos (RR: 5.18; Cl95\%:2.3-11.6) is the author of the abstract and that the abstract was collaboratively written by students from two universities (RR: 3.64 ; CI95\%:1.1-11.7) are associated factors in the multivariate analysis. Conclusions. The publication rate of abstract submitted at national scientific meetings of medical students in Peru is low. New strategies should be taken, and the existing ones should be reinforced in order to increase the publication rate of the abstract submitted.
\end{abstract}

Key words: Research; Congresses; Meeting abstracts; Scientific and technical publications; Medical students (source: $\mathrm{MeSH}$ NLM).

\footnotetext{
Escuela de Medicina, Universidad Peruana de Ciencias Aplicadas. Lima, Perú.

Sociedad Científica de Estudiantes de Medicina de la Universidad Peruana de Ciencias Aplicadas (SOCIEMUPC). Lima, Perú.

Seguro Social de Salud (EsSalud). Lima, Perú.

Estudiante de medicina; ${ }^{\mathrm{b}}$ médico cirujano

Recibido: 23-08-12 Aprobado: 14-11-12

* El presente estudio fue presentado al XXV Congreso Científico Nacional de Estudiantes de Medicina de Perú en Cusco 2011.
}

Citar como: Toro-Polo LM, Pereyra-Elías R, Nizama-Vía A, Ng-Sueng LF, Vélez-Segovia E, Galán-Rodas E, Mayta-Tristán P. Publicación de los trabajos presentados a los congresos científicos de estudiantes de medicina, Perú 2002-2009: características y factores asociados. Rev Peru Med Exp Salud Publica. 2012;29(4):461-8. 


\section{INTRODUCCIÓN}

El desarrollo de un país está relacionado con su capacidad de generar y aplicar el conocimiento que obtuvo a través de la investigación científica (1), aspecto que sucede en todos los ámbitos, incluyendo la Medicina y la salud. El conocimiento científico para que pueda ser utilizado debe ser publicado en revistas científicas, previa evaluación por pares, por lo que se puede afirmar que una investigación no ha culminado hasta que sus resultados sean publicados ${ }^{(2,3)}$.

La investigación es uno de los ejes de la formación médica, y el alumno de Medicina desarrolla varias investigaciones dentro de su malla curricular; adicionalmente, en algunas escuelas de medicina peruanas se requiere sustentar una tesis para obtener el título de médico. Sin embargo, la producción científica peruana en salud es baja ${ }^{(4)}$ y se han planteado medidas para mejorarla, incluyendo entre ellas, el estímulo de la investigación y publicación desde el pregrado para la formación de nuevos investigadores ${ }^{(2,5)}$.

Una de las iniciativas para mejorar la situación de la investigación en pregrado, fue la creación de las sociedades científicas de estudiantes de medicina (SOCEM), las cuales reúnen a estudiantes con vocación científica que se organizan para desarrollar habilidades en investigación ${ }^{(6)}$. A nivel latinoamericano, la entidad que agrupa estas sociedades es la Federación Latinoamericana de Sociedades Científicas de Estudiantes de Medicina (FELSOCEM), la cual anualmente congrega estudiantes de Medicina de toda Latinoamérica, que realizan investigación, con el objetivo de que estos expongan sus trabajos e intercambien experiencias ${ }^{(7)}$. Sin duda alguna, este tipo de evento enriquece el trabajo del estudiante y le permite tener una mejor visión de cómo desarrollar un trabajo de investigación, teniendo como objetivo futuro la publicación. Hasta el año 2010, las SOCEM han demostrado una interesante presencia en la producción de artículos de revistas de Colombia ${ }^{(8)}$, Chile ${ }^{(9)}$, Perú ${ }^{(10)}$ y Venezuela ${ }^{(11)}$ indizadas en SciELO.

En 1992, se creó la Sociedad Científica Peruana de Estudiantes de Medicina (SOCIPEM, ahora Sociedad Científica Médico Estudiantil Peruana - SOCIMEP), la cual organiza los congresos científicos nacionales (CCN) estudiantiles con una presentación aproximada de 60 trabajos por año ${ }^{(12)}$; sin embargo, la producción científica real, evidenciada en revistas peruanas indizadas en SciELO Perú hasta el 2005, no supera los seis artículos originales por año y representa el $4,5 \%$ de los artículos publicados ${ }^{(10)}$, una cifra bastante menor a la de otros países latinoamericanos ${ }^{(8,9)}$; $\sin$ embargo, para el periodo 2009 y 2010 , se ha reportado que el $12,9 \%$ de los artículos originales de revistas médicas indizadas en SciELO Perú han sido publicados por estudiantes de medicina ${ }^{(13)}$.

No se conoce si los trabajos presentados a los congresos de estudiantes de medicina peruanos se publican en revistas científicas, ni qué factores están asociados con esa publicación, aspectos que son los objetivos de esta investigación.

\section{MATERIALES Y MÉTODOS}

\section{DISEÑO Y POBLACIÓN DE ESTUDIO}

Se realizó una cohorte no concurrente en el segundo trimestre del año 2011, para lo cual se revisó la totalidad de los resúmenes de trabajos de investigación presentados a los CCN de de la Sociedad Científica Médico Estudiantil Peruana (SOCIMEP) realizados entre los años 2002 y 2009. Se excluyó los resúmenes presentados por estudiantes de medicina con filiación de una universidad extranjera y los que se presentaron más de una vez (Figura 1).

\section{CARACTERÍSTICAS DE LOS TRABAJOS}

Dos de los autores (EGR y PMT) se encargaron de colectar los libros de resúmenes de los congresos en versión impresa o electrónica, contactándose con los organizadores de cada congreso en caso no hayan participado de estos. Posteriormente, se analizó cada resumen y se generó una base de datos incluyendo la siguiente información: título del trabajo, nombre de los autores y asesores, número de autores, número de asesores, y universidad de filiación de los autores.

Se consignó el área de concurso del resumen, que fueron tres: ciencias básicas, ciencias clínicas y quirúrgicas, o salud pública y educación médica. Se definió a un resumen como "colaborativo" cuando entre los autores del estudio existían alumnos cuyas filiaciones eran de distintas universidades.

Además, se evaluó las características de los resúmenes, dentro de las cuales se incluyó el tipo de estudio clasificado: a) según temporalidad (definido por el momento en que se realizó la recolección de datos): retrospectivos o prospectivos; b) según objetivo: descriptivos o analíticos; c) según intervención: observacionales o experimentales; c) según mediciones: transversales o longitudinales; e) según diseño: serie o reporte de casos, corte transversal, casos y controles, cohortes, experimentales y otros. 


\section{PUBLICACIÓN DE LOS TRABAJOS}

La variable "publicación" fue definida como un artículo publicado en una revista científica que se encuentre indizada en MEDLINE, SciELO, LILACS o RedALyC, o se encuentre disponible en el Portal Revist@s Peruanas o el Sistema de Bibliotecas de la Universidad Nacional Mayor de San Marcos, en el que coincidiese el tiempo, la población de estudio, el resultado principal y al menos un autor con el correspondiente resumen presentado al congreso.

Para determinar que un trabajo presentado al congreso fue publicado, se aplicó una estrategia de búsqueda utilizada previamente para la búsqueda de publicaciones duplicadas ${ }^{(14)}$ y recientemente usada para detectar la publicación de resúmenes de congresos ${ }^{(15)}$. Para ello, con ayuda del buscador avanzado de Google Académico (www.scholar.google.com) se realizó una estrategia de búsqueda usando una combinación de términos que incluya el lugar de estudio, la población de estudio, el resultado principal y tres autores (el apellido paterno del primero, el segundo y el último), de la siguiente forma: ("Lugar de estudio" AND "población de estudio" AND "resultado principal") AND (autor: Primero OR autor: Segundo OR autor: Último). La búsqueda se realizó tanto en español como en inglés. Se seleccionó esta estrategia porque Google Académico permite encontrar artículos y citas de revistas indizadas en diferentes bases de datos, incluyendo todas las que mencionamos en nuestra definición de publicación ${ }^{(16)}$. Cada resumen fue revisado y verificado por dos o más autores para corroborar su publicación entre los meses de junio y julio del año 2011, previa capacitación y estandarización realizada en un trabajo anterior ${ }^{(15)}$, y uno de ellos (PMT) se encargó del control de calidad de la base de datos.

Finalmente, se evaluó las características de los artículos publicados: número de autores, tipo de artículo publicado, el tiempo de diferencia entre su presentación al congreso y su publicación medido en meses, y el año de publicación. También, se evaluó la concordancia entre la información consignada en la publicación con lo que tenía el resumen presentado al congreso, como la concordancia en el número de autores, orden de autores, aparición del asesor, y entre las diferentes secciones del resumen (título, objetivos, conclusiones, etc).

\section{ANÁLISIS DE DATOS}

La base de datos fue generada en el programa Microsoft Excel. Previo control de calidad, los datos fueron ingresados al paquete estadístico STATA 11.0 para su análisis. Se describió las frecuencias y medidas de dispersión central (mediana y rango intercuartílico $[R I Q])$. Para evaluar la asociación entre la publicación

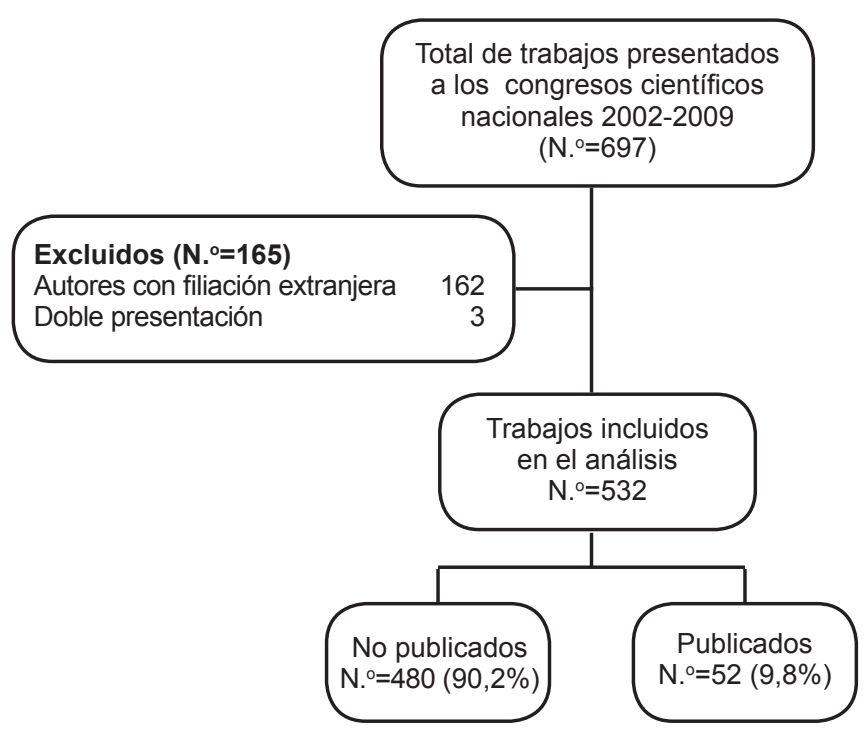

Figura 1. Flujograma de los trabajos presentados a los congresos científicos nacionales, Perú 2002-2009.

de los resúmenes y las variables categóricas se utilizó el chi cuadrado o la prueba exacta de Fisher según correspondiese y para las variables numéricas se usó la U de Mann Withney, previa comprobación de la falta de normalidad con la prueba de Shapiro-Wilk. Para evaluar la asociación entre el tiempo de publicación y las variables numéricas (número de autores, número de asesores) se utilizó la correlación de Spearman. Se calculó los riesgos relativos (RR) con sus intervalos de confianza al 95\% (IC 95\%) tanto a nivel bivariado como multivariado utilizando la regresión de Poisson con varianza robusta. Se consideró un valor de $p<0,05$ como significativo.

\section{RESULTADOS}

De los resúmenes encontrados, se excluyó 162 por tener autores de filiación extranjera y tres por doble presentación (dos de ellos exactamente iguales y un resumen "inflado" al aumentar un año de estudio) (Figura 1).

\section{CARACTERÍSTICAS DE LOS TRABAJOS}

En los ocho años de estudio, se presentaron 135/532 $(25,4 \%)$ resúmenes para el área de ciencias básicas; $157(29,5 \%)$ en el área de ciencias clínicas y quirúrgicas, y $240(45,1 \%)$ en el área de salud pública y educación médica. La mayoría de los estudios fueron de corte transversal $(68,4 \%)$, seguidos por experimentales $(25,4 \%)$, otros diseños utilizados en menor proporción fueron casos y controles, cohortes o series de casos. Según intervención del investigador la mayoría fueron observacionales $(74,6 \%)$, prospectivos $(70,4 \%)$, transversales $(69,3 \%)$ y de diseño analítico $(51,4 \%)$. 
Existen diferencias significativas según el año de congreso para el tipo de estudio según objetivo, con una mayor proporción de estudios analíticos del 2006 al 2009 respecto de años anteriores. No existen diferencias, según año de congreso, para el tipo de estudio según diseño, temporalidad, medición ni intervención $\left(p>0,05 ; X^{2}\right)$.

La mediana de autores de los resúmenes fue de 4,0 (RIQ: 4 a 4) y de asesores 1,0 (RIQ: 1 a 1); no se encontraron diferencias según publicación para estas variables ( $p>0,05, U$ de Mann Withney).

\section{PUBLICACIÓN DE LOS TRABAJOS}

De los 532 resúmenes incluidos, solo 52 (9,8\%) fueron publicados (Figura 1). Existe contraste entre la proporción de publicación en los diferentes años de congreso, variando desde $1 / 42(2,3 \%)$ para el congreso del año 2004 hasta $9 / 55(16,4 \%)$ para el congreso del 2006. Sin embargo, no se encontraron diferencias significativas en el análisis bivariado para la proporción de resúmenes publicados según año de congreso $\left(p>0,05 ; X^{2}\right)$.
La mediana de autores en las publicaciones fue de 5 (RIQ: 4 a 5) y de autores médicos (asesores) de las publicaciones fue 1 (RIQ: 1 a 1 ) y solo en $28 / 41$ resúmenes que tuvieron asesores, estos fueron autores en la publicación. Se encontró diferencias significativas entre el número de autores del resumen presentado al congreso con el número de autores estudiantes en las publicaciones de dichos resúmenes (Wilcoxon; $p<0,01$; diferencia mediana de un autor).

La universidad de filiación de autores con mayor cantidad de resúmenes presentados a congresos y mayor proporción de publicación (representa más del $50 \%$ de los resúmenes publicados) fue la Universidad Nacional Mayor de San Marcos (Tabla 1).

Al evaluar concordancia entre el resumen y la publicación, se observó que el título del resumen solo se mantuvo en 16 publicaciones. En 26 publicaciones hubo autores distintos a los del resumen; mientras que el orden de todos los autores permaneció igual en 26 artículos y el primer autor fue el mismo en 45 . Asimismo, hubo 43

Tabla 1. Trabajos presentados a los congresos científicos nacionales de SOCIMEP según universidad, frecuencia y publicación.

\begin{tabular}{|c|c|c|c|c|c|}
\hline \multirow[b]{2}{*}{ Universidad } & \multirow[b]{2}{*}{ Ciudad } & \multirow[b]{2}{*}{ Tipo } & \multicolumn{3}{|c|}{ Resúmenes presentados } \\
\hline & & & Total & $\begin{array}{l}\text { Media por } \\
\text { año }\end{array}$ & $\begin{array}{c}\text { Publicados } \\
(\%)\end{array}$ \\
\hline Universidad Nacional Mayor de San Marcos & Lima & Pública & 100 & 12,5 & $30(30)$ \\
\hline Universidad Nacional Federico Villareal & Lima & Pública & 62 & 7,8 & $3(4,8)$ \\
\hline Universidad de San Martín de Porres & Lima & Privada & 52 & 6,5 & $3(5,8)$ \\
\hline Universidad Nacional de Trujillo & Trujillo & Privada & 39 & 4,9 & $3(7,7)$ \\
\hline Universidad Nacional San Luis Gonzaga & Ica & Pública & 32 & 4,0 & $3(9,4)$ \\
\hline Universidad Nacional de San Antonio Abad & Cusco & Pública & 32 & 4,0 & $1(3,1)$ \\
\hline Universidad Ricardo Palma & Lima & Privada & 26 & 3,3 & $1(3,8)$ \\
\hline Universidad Nacional del Altiplano & Puno & Pública & 23 & 2,9 & $1(4,3)$ \\
\hline Universidad Nacional del Centro del Perú & Huancayo & Pública & 20 & 2,5 & 0 \\
\hline Universidad Nacional de San Agustín & Arequipa & Pública & 20 & 2,5 & $2(10)$ \\
\hline Universidad Nacional Pedro Ruiz Gallo & Chiclayo & Pública & 14 & 1,8 & 0 \\
\hline Universidad Nacional José Faustino Sánchez Carrión & Huacho & Pública & 13 & 1,6 & $3(23,1)$ \\
\hline Universidad Nacional de Piura & Piura & Pública & 13 & 1,6 & $1(7,7)$ \\
\hline Universidad Nacional de la Amazonía Peruana & Iquitos & Pública & 10 & 1,3 & 0 \\
\hline Universidad Privada Antenor Orrego & Trujillo & Privada & 12 & 1,5 & 0 \\
\hline Universidad Privada Los Andes & Huancayo & Privada & 12 & 1,5 & $1(8,3)$ \\
\hline Universidad Nacional Jorge Basadre Grohmann & Tacna & Pública & 9 & 1,1 & $1(11,1)$ \\
\hline Universidad Católica Santa María & Arequipa & Privada & 10 & 1,0 & $1(10)$ \\
\hline Universidad Nacional de Cajamarca & Cajamarca & Pública & 8 & 1,0 & $1(12,5)$ \\
\hline Universidad de Chiclayo & Chiclayo & Privada & 8 & 1,0 & 0 \\
\hline Universidad Peruana Cayetano Heredia & Lima & Privada & 6 & 0,8 & 0 \\
\hline Universidad Privada San Pedro & Chimbote & Privada & 4 & 0,5 & 0 \\
\hline Asociación Universidad Privada San Juan Bautista & Lima & Privada & 4 & 0,5 & 0 \\
\hline Universidad Privada de Tacna & Tacna & Privada & 3 & 0,4 & 0 \\
\hline Universidad Nacional de Ucayali & Pucallpa & Pública & 2 & 0,3 & 0 \\
\hline Universidad Nacional Hermilio Valdizán & Huánuco & Pública & 2 & 0,3 & 0 \\
\hline Colaborativo & & & 6 & 0,8 & $3(50)$ \\
\hline Total & & & $542^{*}$ & 66,1 & $52(9,8)$ \\
\hline
\end{tabular}


Tabla 2. Publicaciones de los trabajos presentados a congresos científicos estudiantiles peruanos 2002-2009: revista de destino y tipo de artículo.

\begin{tabular}{|c|c|c|c|c|c|c|c|}
\hline \multirow[b]{2}{*}{ Revista } & \multirow[b]{2}{*}{ N. ${ }^{\circ}$} & \multicolumn{3}{|c|}{ Tipo de artículo } & \multicolumn{3}{|c|}{ Indización } \\
\hline & & Original & $\begin{array}{c}\text { Original } \\
\text { Breve }\end{array}$ & Carta & MEDLINE & SCIELO & LILACS \\
\hline CIMEL & 13 & 13 & 0 & 0 & No & Sít & No \\
\hline Anales de la Facultad de Medicina & 10 & 9 & 1 & 0 & No & Sí & Sí \\
\hline Rev Peru Med Exp Salud Publica & 10 & 8 & 0 & 2 & $\mathrm{Si}^{* *}$ & Sí & Sí \\
\hline Acta Médica Peruana & 4 & 3 & 1 & 0 & No & Sí & Sí \\
\hline Revista Horizonte Médico & 3 & 3 & 0 & 0 & No & No & No \\
\hline Revista Peruana de Epidemiología & 3 & 2 & 0 & 1 & No & No & No \\
\hline Folia Dermatológica Peruana & 2 & 2 & 0 & 0 & No & Sít & Sí \\
\hline Revista de Gastroenterología del Perú & 1 & 1 & 0 & 0 & Sí & Sí & Sí \\
\hline Revista Médica Herediana & 1 & 1 & 0 & 0 & No & Sí & Sí \\
\hline Diagnóstico & 1 & 1 & 0 & 0 & No & No & Sí \\
\hline Dermatología Peruana & 1 & 1 & 0 & 0 & No & Sí & Sí \\
\hline Revista Peruana de Ginecología y Obstetricia & 1 & 1 & 0 & 0 & No & No & No \\
\hline Revista de la Sociedad Peruana de Neumología & 1 & 1 & 0 & 0 & No & No & No \\
\hline Revista del Encuentro Científico Nacional & 1 & 1 & 0 & 0 & No & No & No \\
\hline
\end{tabular}

* Todas las publicaciones se realizaron en revistas peruanas, en idioma español y sin indización al Science Citation Index Expanded.

** Esta revista se encuentra indizada en Medline desde enero de 2010.

$\dagger$ Esta revista fue retirada de SciELO en noviembre de 2009.

concordancias en cuanto a objetivos principales del resumen y la publicación y 42 concordancias respecto de los resultados principales del estudio.

El tiempo medio de diferencia entre el congreso y la publicación fue de 22,6 $\pm 24,0$ meses (mediana de 13 , rango 0 a 75 ). No se encontró asociación entre el tiempo de publicación y el tipo de estudio según mediciones, objetivo, intervención y diseño, ni según la concordancia de número de autores, orden de los autores, primer autor y resultados principales $(U$ de Mann Whitney; $p>0,05)$, así como con el número de autores $o$ asesores (Spearman; $p>0,05$ ). Los estudios prospectivos se publicaron en menor tiempo (21 meses) que los retrospectivos ( $p=0,007)$, y aquellos en los que hubo concordancia entre los objetivos del resumen y del artículo también se publicaron más rápido (22 meses; $p=0,008)$.

Tabla 3. Factores asociados con la publicación de los trabajos presentados a los congresos científicos nacionales de SOCIMEP, 2002-2009.

\begin{tabular}{|c|c|c|c|c|c|c|c|c|}
\hline \multirow{3}{*}{ Características } & \multicolumn{2}{|c|}{ Publicado } & \multicolumn{3}{|c|}{ Análisis bivariado } & \multicolumn{3}{|c|}{ Análisis multivariado† } \\
\hline & Sí & No & RR & (IC95\%) & $\mathrm{p}$ & $\mathbf{R R}$ & (IC95\%) & $\mathrm{n}$ \\
\hline & N. ${ }^{\circ}(\%)$ & N. ${ }^{\circ}(\%)$ & & $(11490 \%)$ & p & & (IC95\%) & $p$ \\
\hline \multicolumn{9}{|c|}{ Autor estudiante de la UNMSM } \\
\hline Sí & $30(30,0)$ & $70(70,0)$ & 5,9 & $(3,5-9,7)$ & $<0,001$ & 5,2 & $(2,3-11,6)$ & $<0,001$ \\
\hline No & $22(5,1)$ & $408(94,9)$ & 1,0 & & & 1,0 & & \\
\hline \multicolumn{9}{|l|}{ Autoría colaborativa } \\
\hline Sí & $3(50,0)$ & $3(50,0)$ & 5,4 & $(2,3-12,5)$ & $<0,001$ & 3,6 & $(1,1-11,7)$ & 0,030 \\
\hline No & $49(9,3)$ & $477(90,7)$ & 1,0 & & & 1,0 & & \\
\hline \multicolumn{9}{|c|}{ Autor de universidad nacional* } \\
\hline Sí & $48(12,0)$ & $351(88,0)$ & 3,9 & $(1,4-10,7)$ & 0,007 & 1,7 & $(0,6-4,7)$ & 0,310 \\
\hline No & $4(3,0)$ & $127(97,0)$ & 1,0 & & & 1,0 & & \\
\hline \multicolumn{9}{|c|}{ Tipo de estudio según objetivo } \\
\hline Analítico & $34(12,5)$ & $238(87,5)$ & 1,8 & $(1,0-3,1)$ & 0,034 & 1,4 & $(0,8-2,5)$ & 0,191 \\
\hline Descriptivo & $18(6,9)$ & $242(93,1)$ & 1,0 & & & 1,0 & & \\
\hline \multicolumn{9}{|c|}{ Autores de universidades de Lima } \\
\hline Sí & $37(14,8)$ & $213(85,2)$ & 2,8 & $(1,6-4,9)$ & 0,001 & 1,0 & $(0,4-2,2)$ & 0,928 \\
\hline No & $15(5,4)$ & $265(94,6)$ & 1,0 & & & 1,0 & & \\
\hline \multicolumn{9}{|l|}{ Año de congreso $\ddagger$} \\
\hline 2006-2009 & $31(11,7)$ & $247(92,2)$ & 1,5 & $(0,9-2,5)$ & 0,133 & 1,6 & $(0,9-2,7)$ & 0,086 \\
\hline $2002-2005$ & $21(7,8)$ & $233(88,3)$ & 1,0 & & & 1,0 & & \\
\hline
\end{tabular}


La principal revista destino de publicación fue CIMEL $13 / 52$ (25,0\%). Todas las revistas donde se publicaron los resúmenes fueron peruanas y en español. Se publicaron 47 artículos originales, dos originales breves y tres cartas al editor (Tabla 2).

No se encontró asociación entre la publicación con el tipo de estudio según diseño ni con el área de concurso $\left(X^{2} ; p>0,05\right)$. En el análisis multivariado final, se encontró asociación con que el autor del resumen sea alumno de la Universidad Nacional Mayor de San Marcos y haber sido un resumen de autoría colaborativa entre más de una universidad (Tabla 3).

\section{DISCUSIÓN}

La publicación de resúmenes de trabajos de investigación presentados en los Congresos Científicos Nacionales (CCN) de SOCIMEP entre los años 2002 y 2009 representa un bajo porcentaje sobre el total de trabajos enviados. Uno de los principales motivos que explicaría esta situación es que, en el Perú, la producción científica se caracteriza por ser escasa ${ }^{(4)}$ y la cultura de publicación de trabajos de investigación es muy pobre ${ }^{(5)}$. Esto se ve reflejado en que el país aporta menos del $0,1 \%$ de artículos a nivel mundial, de los cuales $32,2 \%$ son artículos del área de medicina; además, contribuye con $0,95 \%$ de los artículos latinoamericanos, de los cuales el $78 \%$ se realiza con participación de autores extranjeros ${ }^{(4,17)}$.

Comparativamente, los artículos publicados a partir de los trabajos presentados a congresos peruanos es baja en comparación a lo que sucede en Brasil, en donde la proporción de publicación de los congresos de estudiantes de medicina fue del 33,8\% (18). Asimismo, la proporción de publicación encontrada en nuestro estudio es notablemente menor comparada con el promedio mundial de publicación de investigaciones presentadas en congresos que, según una revisión sistemática del 2007, se encuentra en $44 \%{ }^{(19)}$. A pesar de esto, el porcentaje de publicación de trabajos presentados en los congresos estudiantiles fue superior al porcentaje de publicaciones de los congresos de gastroenterología en Perú entre los años 2002-2008 (15). Esto se puede explicar por la deficiente cultura de investigación y publicación ya mencionada, tanto en la enseñanza de pregrado y posgrado ${ }^{(4,5,20)}$. Esta mayor proporción de publicación, puede deberse a las distintas estrategias desarrolladas en el pregrado, la que incluye cursos extracurriculares, asesoría e impulso por parte de las sociedades científicas estudiantiles ${ }^{(21-23)}$.

Por otro lado, un factor relacionado con la no publicación podría ser la calidad de los resúmenes presentados. Si bien esta no ha sido medida objetivamente, la proporción de publicación de los resúmenes ha sido tomada por algunos autores como un indicador de la calidad de estos y, en consecuencia, del certamen ${ }^{(24)}$.

De los resúmenes enviados a los Congresos Científicos Nacionales de SOCIMEP, la Universidad Nacional Mayor de San Marcos (UNMSM) es responsable de casi el $20 \%$, lo cual la convierte en la universidad con el número más alto de trabajos enviados y también de artículos publicados. La UNMSM tiene la mayor proporción de créditos académicos de cursos de investigación de las universidades peruanas ${ }^{(25)}$, lo que podría explicar esta diferencia tanto en producción de trabajos como en su publicación. Estos resultados confirman lo encontrado previamente en publicaciones de revistas SciELO Perú, donde la UNMSM aportó el $41 \%$ de las publicaciones estudiantiles ${ }^{(7)}$.

Por otro lado, es importante señalar que la Universidad Peruana Cayetano Heredia, institución dedicada a la investigación en el Perú ${ }^{(4)}$, ha mantenido un relevante aporte estudiantil en el área de investigación médica, por lo que ha sido señalada como la de mayor producción científica a nivel nacional entre los años 2009 y 2010 , llegando a representar el $34 \%$ del total de publicaciones ${ }^{(13)}$. Sin embargo, este hecho no se refleja en el presente estudio debido a que la participación de dicha universidad en los CCN es escasa.

Con relación al tiempo de publicación, se estima que el tiempo que transcurre entre el envío del resumen a un congreso y su publicación como artículo en una revista indizada se encuentra alrededor de los dos años, y raramente supera los tres años ${ }^{(26)}$. El periodo de 22,6 meses para la publicación de los artículos presentados en los CCN es ligeramente menor o, inclusive, similar al promedio y al reportado en anteriores investigaciones; sin embargo, presenta una dispersión mucho mayor a la observada en dichas experiencias (26-28). Otro estudio peruano presenta un tiempo de publicación considerablemente menor ${ }^{(15)}$. Estas variaciones en el tiempo de publicación pueden ser explicadas, en parte, por la mayor rapidez de aceptación y por un proceso de revisión más asequible de las revistas en las que se han publicado los estudios evaluados (todas peruanas). Además, estas revistas suelen publicarse a destiempo (artículos se publican en un número correspondiente a meses o años previos al momento actual).

En cuanto a los factores asociados con la publicación de artículos, encontramos que si el autor es alumno de la UNMSM estaba asociado con una mayor probabilidad de publicación. La Sociedad Científica de San Fernando (SOCEM de la UNMSM) presenta una producción científica por encima de la mayoría de universidades 
representando desde entonces poco más de la quinta parte de la producción científica nacional, seguida, en orden, por la Universidad Nacional San Luis Gonzaga, Universidad San Martín de Porres y la Universidad Nacional San Agustín de Arequipa ${ }^{(12)}$, lo cual es similar a lo encontrado en el presente estudio. Además, la Facultad de Medicina de la UNMSM cuenta con una de las pocas revistas científicas que son publicadas con regularidad y que se encuentran indizadas en SciELO-Perú, lo que sugiere una fuerte cultura de publicación dentro de la institución por parte de alumnos y docentes ${ }^{(10)}$ y tienen a la sede editorial de la revista CIMEL, que es la revista científica estudiantil de la FELSOCEM.

Se encontró también que haber realizado un trabajo en conjunto entre dos o más universidades aumenta en gran medida las probabilidades de publicación. Este resultado muestra que la cooperación entre investigadores impulsa la conclusión de un proyecto y eleva el nivel científico de las investigaciones (29,30); por ello se recomienda la investigación en redes y en forma colaborativa, ya que permite obtener lo mejor de cada miembro de la red para generar un mejor trabajo de investigación.

Encontramos diferencias entre el número de autores que aparecían en el trabajo presentado al congreso y el que apareció en el artículo publicado, más que un reflejo de faltas éticas en publicación (que podrían ocurrir) esta situación puede explicarse porque los organizadores de algunos congresos estudiantiles han puesto un límite en el número de autores de 5 o 6 estudiantes o de 1 o 2 médicos, que al lograrse la publicación se sincera el número real de autores.

Nuestro estudio presenta ciertas limitaciones. La mala calidad de algunos de los resúmenes, en donde no se especifica el tipo de estudio y la descripción de materiales y métodos no es clara, lo cual no permitió una óptima clasificación de algunos de ellos. Por otro lado, como se mencionó anteriormente, las revistas peruanas se publican de forma anacrónica, lo cual no permite determinar con exactitud el verdadero tiempo de publicación de los resúmenes, motivo por el cual no consideramos pertinente realizar curvas tipo sobrevida o estimar los hazard ratio. Otra limitación supone no haber obtenido directamente de los autores las razones de no publicación; futuros estudios podrían evaluar este aspecto. Adicionalmente, a partir del 2007, se implementó con mayor énfasis las estrategias de promoción de investigación y publicación en pregrado ${ }^{(6)}$, por lo que sería importante ampliar los años de estudio y evaluar su impacto.

Se recomienda implementar un riguroso proceso de selección de los resúmenes presentados a los Congresos Científicos Nacionales de SOCIMEP antes de su aceptación, realizado por expertos en metodología de la investigación y bioestadística. Ya que una posibilidad de su no publicación puede radicar en que se aceptan trabajos a los CCN de dudosa calidad, con ello, se incrementaría la calidad de los trabajos aceptados así como su posibilidad de publicación, cumpliendo con el objetivo último de la investigación y de las sociedades científicas.

Concluimos que existe una baja proporción de publicación de los resúmenes enviados a los Congresos Científicos Nacionales de SOCIMEP y que la publicación de estos resúmenes se encuentra asociada a que el autor sea alumno de la Universidad Nacional Mayor de San Marcos y que el estudio haya sido desarrollado de forma colaborativa por autores de mas de dos universidades.

Contribuciones de autoría: LMTP, PMT, RPE, ANV participaron en la concepción y diseño del estudio. EGR y PMT recopilaron los libros de resúmenes. LMTP, RPE, ANV, LFNS y EVS recopilaron los datos; PMT realizó el control de calidad y el análisis de datos. LMTP, RPE y PMT redactaron el manuscrito; ANV, EGR, EVS, LFNS aportaron críticamente al manuscrito. Todos los autores aprobaron la versión final del trabajo.

Fuentes de financiamiento: autofinanciado.

Conflictos de interés: PMT fue alumno de la Universidad Nacional Mayor de San Marcos y es exmiembro y asesor de la Sociedad Científica de San Fernando.

\section{REFERENCIAS BIBLIOGRÁFICAS}

1. King DA. The scientific impact of nations. Nature. 2004;430(6997):311-6.

2. Huamaní C, Mayta-Tristán P, Rodríguez-Morales AJ. Publicar desde pregrado. Interciencia. 2008;33(11):785.

3. Rojas-Revoredo V. Las publicaciones en revistas indexadas, único indicador de la producción de las sociedades científicas estudiantiles. CIMEL. 2007;12(1):5-6
4. Huamani C, Mayta-Tristán P. Producción científica peruana en medicina y redes de colaboración, análisis del Science Citation Index 2000-2009. Rev Peru Med Exp Salud Publica. 2010;27(3):315-25.

5. Yagui M, Espinoza M, Caballero P, Castilla T, Garro G, Yamaguchi LP, et al. Avances y retos en la construcción del sistema nacional de investigación en salud en el Perú. Rev Peru Med Exp Salud Publica. 2010;27(3):387-97.

6. Huamaní C, Chávez-Solís P, Domínguez-Haro, Solano-Aldana M. Producción científica estudiantil: análisis y expectativa. Rev Peru Med Exp Salud Publica. 2007;24(4):444-46.

7. Oróstegui-Panilla D, Cabrera-Samith I, Angulo-Bazán Y, Mayta-Tristán P, Rodríguez-Morales AJ. Encuentros in- 
ternacionales de revistas científicas estudiantiles de las ciencias de la salud en Latinoamérica, 1998-2008. Rev Panam Salud Publica. 2009;25(5):469-70.

8. Pachajoa-Londoño HM. Publicación de artículos originales desde el pregrado en una revista médica colombiana entre 1994-2004. CIMEL. 2006;11(1):24-6.

9. Poggi-Sanz P, Huamaní C, ChávezSolís P, Mayta-Tristán P. Participación de estudiantes de pregrado en la publicación de artículos en revistas médicas chilenas 2001-2005. Libro de resúmenes: XX Congreso Científico Nacional de Estudiantes de Medicina de Perú. Cajamarca, Perú, 2006.

10. Huamaní C, Chávez-Solis P, MaytaTristán P. Aporte estudiantil en la publicación de artículos científicos en revistas médicas indizadas en SciELOPerú, 1997-2005. An Fac Med (Lima). 2007;69:42-5.

11. Angulo R, Angulo F, Huamaní C, Mayta-Tristán P. Publicación estudiantil en revistas médicas venezolanas 20012005. CIMEL. 2008;13:6-8.

12. Galán E, Manrique N, Villavicencio E, Yllatopa E, Peralta M, De la Cruz W. Producción científica de los investigadores del pregrado de medicina humana del Perú, 1993-2003. CIMEL. 2005;10(1):41-8.

13. Taype-Rondán A, Lajo-Aurazo Y, Gutiérrez-Brown R, Zamalloa-Masías N, Saldaña-Gonzales M. Aporte de las sociedades estudiantiles en la publicación científica en SciELO-Perú, 2009-2010. Rev Peru Med Exp Salud Publica. 2011;28(4):691-2.

14. Mayta-Tristán P, Mezones-Holguin E. Aclaración editorial. Rev Peru Med Exp Salud Publica. 2009;26(3):411-2.

15. Pereyra-Elías R, Ng-Sueng LF, ToroPolo LM, Nizama-Vía A, Piscoya A, Mayta-Tristán P. Baja publicación de los trabajos presentados a los Congresos de la Sociedad de Gastroenterología del
Perú 1998-2008. Rev Gastroenterol Perú. 2011;31(2):124-32.

16. Huamani C, Pacheco-Romero J. Visibilidad y producción de las revistas peruanas. Rev Gastroenterol Perú. 2009;29(2):132-9.

17. SCImago Internet]. Granada: ScimagoLab; 2011 [citado el 15 julio del 2011]. Disponible en: http://www. scimagojr.com

18. Fede AB, Miranda MC, Lera AT, Ueda A, Antonangelo DV, Schaffhausser $\mathrm{HL}$, et al. Experience with the $\mathrm{ABC}$ Foundation School of Medicine undergraduate meeting. Rev Assoc Med Bras. 2010;56(3):313-7.

19. Scherer RW, Langenberg P, von Elm E. Full publication of results initially presented in abstracts. Cochrane Database Syst Rev. 2007;(2):MR000005.

20. Molina-Ordóñez J, Huamaní C, Mayta-Tristán P. Apreciación estudiantil sobre la capacitación universitaria en investigación: Estudio preliminar. Rev Peru Med Exp Salud Publica. 2008;25(3):325-9.

21. Mayta-Tristán P. Sociedades científicas de estudiantes de medicina: el futuro de la investigación en Latinoamérica. MedUNAB. 2010;13(1):3-4.

22. Mayta-Tristán P, Peña-Oscuvilca A. Importancia de la publicación en las sociedades científicas de estudiantes de medicina del Perú: estudio preliminar. CIMEL. 2009; 14(1):27-34.

23. Pereyra-Elías R, Rodríguez-Morales A, Mayta-Tristán P. Undergraduate publication in Latin America: Role of medical student's scientific societies. Med Teach. 2011;33(7):594.

24. Macmillan CD, Moore AK, Cook RJ, Pedley DK. Abstract-to-publication ratio for papers presented at scientific meetings: a quality marker for UK emergency medicine research. Emerg Med J. 2007;24(6):425-6.
25. Díaz-Vélez C, Manrique-González LM, Galán-Rodas, E, Apolaya-Segura M. Conocimientos, actitudes y prácticas en investigación de los estudiantes de pregrado de facultades de medicina del Perú. Acta Med Peru. 2008;25(1):9-15.

26. Timmer A, Blum T, Lankisch PG. Publication rates following pancreas meetings. Pancreas. 2001;23(2):212-5.

27. Cifuentes I, Bartolí Solé R, Miquel Planas M, Cabré Gelad E, Morillas Cunill RM, Planas Vila R. ¿Cuántos alcanzan la meta? Destino de los resúmenes presentados en el XXIV Congreso de la Asociación Española para el Estudio del Hígado y su evolución en el tiempo. Gastroenterol Hepatol. 2007;30(5):263-7.

28. Timmer A, Hilsden RJ, Cole J, Hailey $\mathrm{D}$, Sutherland LR. Publication bias in gastroenterological research - a retrospective cohort study based on abstracts submitted to a scientific meeting. BMC Med Res Methodol. 2002;2:7.

29. Haines VA, Godley J, Hawe P. Understanding interdisciplinary collaboration as social networks. Am J Community Psychol. 2011;47(1-2):1-11.

30. Morel CM, Serruya SJ, Penna GO, Guimaraes R. Co-authorship analysis: a powerful tool for strategic planning of research, development and capacity building programs on neglected diseases. PLoS Negl Trop Dis. 2009;3(8):e501.

Correspondencia: Percy Mayta-Tristán Dirección: Av. Brasil 2169, Dpto 802, Lima 11, Perú.

Teléfono: (511) 313-3333 anexo 2727

Correoelectrónico:percy.mayta@upc.edu.pe 